\title{
Quantificação de células CD 34+ em sangue periférico, produto de aférese e cordão umbilical: estudo comparativo de três diferentes metodologias
}

\author{
Nydia S. Bacal \\ João C. C. Guerra \\ Euripedes Ferreira \\ Sonia T. Nozawa \\ Ruth H. Kanayama \\ Daniel Di Pietro \\ Jacyr Pasternack \\ Nelson Hamerschlack \\ Luiz G. M. Rosenfeld
}

\begin{abstract}
A quantificação das células CD34+ em sangue periférico é utilizada para determinar o melhor momento de iniciar a aférese, enquanto que na leucoaférese e no sangue de cordão umbilical determinam a quantidade de células CD34+ para o transplante de células progenitoras. O objetivo deste trabalho foi comparar três diferentes metodologias de quantificação de células CD34+. Foram utilizados três diferentes tipos de amostras: a) 32 amostras de sangue periférico, coletadas de pacientes estimulados com G-CSF $50 \mu \mathrm{g} / \mathrm{Kg} /$ dose total, sem quimioterapia na mobilização. b) 31 amostras de produto de aférese de pacientes estimulados com o mesmo protocolo de G-CSF. c) 20 amostras de sangue de cordão coletadas em CPDA-1, por punção de veia umbilical. As amostras permaneceram à temperatura ambiente no máximo até $24 h$ da análise. O citômetro de fluxo utilizado foi o Epics XL-MCL (Coulter) com os protocolos de dupla plataforma: ISHAGE e Mulhouse modificado para análise de maior numero de eventos, e o citômetro Imagn 2000 (Biometric Imaging) de plataforma única, conforme técnicas recomendadas. Os anticorpos monoclonais utilizados foram: CD45FITC, CD34-PE, e isotipo IgG1-PE da Immunotech. As análises estatísticas foram: ANOVA e correlação de teste $t$ de Student. Os resultados não apresentaram diferenças estatisticamente significativas nos três métodos.
\end{abstract}

Rev.bras.hematol.hemoter., 2001, 23 (2): 69-78

Palavras-chave: Quantificação de CD34, citometria de fluxo, citometria com volume fixo, plataforma

\section{Introdução}

As células marcadas com anticorpo monoclonal CD34 representam as células hematopoéticas tronco (stem cell). Sua concentração no sangue periférico determinada por Citômetro de Fluxo ou Citômetro de volume fixo oscila entre 0,01 a $0,05 \%$ (1); a concentração na medula óssea é de $<1 \%$ das células mononucleares normais (2) e no sangue de cordão umbilical varia de 0,33 a $1,98 \%(3,4,5)$.

É reconhecido que estas células tronco infundidas em um indivíduo após a quimioterapia e/ou radioterapia ablativas da medula óssea, instalam-se na medula óssea, sendo responsáveis pela regeneração hematopoética. Os métodos para obtenção de células tronco periféricas vão desde a coleta de medula óssea de doadores; coleta por leucoaférese do paciente ou doador após mobilização com estímulo com fator de crescimento (G-CSF) associado ou não a quimioterapia prévia em casos de pacientes, até a utilização de sangue de cordão umbilical. Experimentalmente métodos de concentração e de expansão in vitro vêm sendo desenvolvidos.

As concentrações obtidas nas leucoaféreses apresentam correlação direta com a quantificação do CD 34 no sangue periférico $(6,7,8,9,10)$, mas os resultados finais obtidos do produto total das leucoaféreses por paciente são extremamente variáveis, como demonstrado na publicação do Centro Médico da Universidade de Chicago (6).

Laboratório Clinico do Hospital Albert Einstein

Correspondência: Nydia Strachman Bacal

Hospital Albert Einstein. Av. Albert Einstein 627 - $4^{\circ}$ andar. Laboratório de Patologia Clínica.

05652-000. São Paulo. SP.

Fone: (11) 3747-0450. Fax: (11) 3747-0616 
Nessa análise de 514 produtos de aférese preparados de 100 pacientes, a concentração final obtida varia de 0,04 a 58,08 células CD34+ x 10 por quilo de peso com uma média de 1,87.

A quantidade de células CD34 positivas infundidas para fins de recuperação da hematopoese tem sido relacionada à viabilidade do procedimento e à velocidade de recuperação hematopoética (11). A quantidade mínima recomendada por vários serviços é de $2 \times 10^{6}$ células/Kg de peso do receptor, porém existem grandes diferenças na literatura que vão desde $1 \mathrm{a}$ $2,5(12,13,14)$ e não tem sido possível demonstrar estatisticamente a correlação numérica entre o número de células infundidas e o número de dias para recuperação de parâmetros hematológicos específicos. Este fato tem sido atribuído a heterogeneidade dos métodos de quantificação de células CD34 positivas e a variabilidade da própria metodologia $(15,16)$.

O objetivo deste trabalho é comparar três diferentes metodologias de quantificação de células CD34+ em diferentes materiais biológicos, analisando as diferenças entre os resultados obtidos e sua aplicação nos processos de recuperação da medula óssea por infusão de células progenitoras hematopoéticas.

\section{Casuística e Método}

As amostras testadas foram obtidas no Banco de Sangue do Hospital Albert Einstein de São Paulo, de pacientes submetidos a quimioterapia e ou radioterapia ablativas em procedimentos de recuperação hematopoética. Foram observadas 32 amostras de sangue periférico, colhidas em EDTA, de pacientes estimulados com G-CSF na dose de $50 \mathrm{mcg} / \mathrm{Kg}$, sem quimioterapia prévia; 31 amostras de leucoaférese colhidas em ACD após processamento do volume médio de 10 litros de sangue à velocidade de 40 a $80 \mathrm{ml} / \mathrm{min}$ na Processadora CS 3000 (Baxter) e 20 amostras de sangue de cordão umbilical colhidos em CPDA-1 e analisados antes de seu congelamento. Os produtos de aférese, devido ao alto número de leucócitos, foram diluídos para obter aproximadamente $100 \times 10^{9}$ leucócitos/dl com tampão de PBS com 1\% de albumina bovina. Todos os materiais foram mantidos à temperatura ambiente e analisados para determinação de células CD34 positivas nas três metodologias antes de 24 horas de suas obtenções.

Os métodos utilizados na contagem de células CD34+ foram a citometria de fluxo em citômetro EPICS XL (Coulter) seguindo dois diferentes protocolos (ISHAGE e Mulhouse Mod. por Salamanca) e o citômetro de volume fixo Imagn 2000 (Biometric Imaging). Para a citometria de fluxo os materiais foram submetidos à hemólise e fixação pela metodologia da Coulter Q-Prep, de acordo com as recomendações do fabricante. A concentração absoluta de leucócitos de cada material foi obtida em outra plataforma, Coulter T890. Os protocolos das estratégias de quantificação do percentual de células CD34 positivas no citômetro de fluxo adotados foram segundo as descritas por Sutherland - ISHAGE (17) e o protocolo de Mulhouse modificado pelo aumento do número de eventos analisados $(18,19)$.

As determinações no citômetro de volume fixo Imagn 2000 foram realizadas em plataforma única, obtendo-se diretamente o número absoluto de células CD34 positivas utilizando os reagentes e método do fabricante (20).

Os anticorpos monoclonais utilizados foram fornecidos pela Immunotech: CD45, clone J33-FITC; CD34, clone 581-PE com controle isotipo IgG1-PE de camundongo.

Os métodos de determinação de CD34 em citometria de fluxo por ambos os protocolos vêm sendo utilizados em nosso Laboratório há mais de quatro anos com coeficiente de variação determinado em duplicatas no sistema de controle de qualidade interno inferior a $15 \%$ e nos dois últimos anos nossos resultados têm sido aferidos pela participação no sistema de controle de qualidade externo (UK NEQAS - United Kingdom) com resultados dentro dos limites aceitos, ou seja, inferiores a $25 \%$ de variação interlaboratorial (21).

Os resultados foram analisados através de análise estatística informatizada utilizando o teste T de Student para os dados paramétricos, e o de Mann Whitney para os não paramétricos.

\section{Resultados}

Visando a avaliação intrínseca das metodologias foram realizadas 10 determinações em uma amostra de sangue periférico para cada um dos métodos e os resultados obtidos estão descritos na Tabela 1.

Os resultados nas 32 amostras de Sangue Periférico colhidos após mobilização com G-CSF estão descritos na Tabela 2.

Os resultados nas 31 amostras de Produto de Aférese, colhidos após mobilização com G-CSF estão descritos na Tabela 3.

Os resultados nas 20 amostras de Sangue de Cordão Umbilical estão descritos na Tabela 4. 
Tabela 1. Avaliação de precisão das metodologias

\begin{tabular}{l|c|c}
\hline Sangue Periférico & $\begin{array}{l}\text { Média CD34(+) } \\
\text { células/mm }\end{array}$ & $\begin{array}{c}\text { Coeficiente de } \\
\text { Variação }\end{array}$ \\
\hline $\begin{array}{l}\text { Amostra 1 } \\
\text { Método: Imagn }\end{array}$ & 35 & $11 \%$ \\
$\begin{array}{l}\text { Amostra 2 } \\
\text { Método: Salamanca }\end{array}$ & 26 & $11 \%$ \\
$\begin{array}{l}\text { Amostra 3 } \\
\text { Método: ISHAGE }\end{array}$ & 15 & $15 \%$ \\
\hline
\end{tabular}

Tabela 2. Concentração absoluta de células CD34+ em amostras de sangue periférico pelos três métodos de análise

\begin{tabular}{|c|c|c|c|}
\hline $\begin{array}{l}\text { Identificação } \\
\text { Pac./no Afererese }\end{array}$ & $\begin{array}{l}\text { P. Mulhouse mod. } \\
\text { células } / \mathrm{mm}^{3}\end{array}$ & $\begin{array}{l}\text { Prot. ISHAGE } \\
\text { células } / \mathrm{mm}^{3}\end{array}$ & $\begin{array}{l}\text { Imagn } 2000 \\
\text { células } / \mathrm{mm}^{3}\end{array}$ \\
\hline ARCM.1 & 15 & 5 & 9 \\
\hline ARCM.2 & 16 & 16 & 13 \\
\hline MLB & 14 & 11 & 15 \\
\hline FIT & 34 & 21 & 23 \\
\hline SDCM & 13 & 5 & 13 \\
\hline MGC.1 & 2 & 0 & 7 \\
\hline MGC.2 & 7 & 0 & 6 \\
\hline MGC.3 & 4 & 2 & 6 \\
\hline MGC.4 & 36 & 20 & 30 \\
\hline MGC. 5 & 21 & 21 & 13 \\
\hline AGF.1 & 4 & 5 & 7 \\
\hline AGF.2 & 17 & 2 & 16 \\
\hline AGF.3 & 3 & 0 & 8 \\
\hline EB.1 & 5 & 0 & 9 \\
\hline EB.2 & 4 & 0 & 5 \\
\hline EB.3 & 5 & 0 & 5 \\
\hline LS.1 & 3 & 3 & 3 \\
\hline LS. 2 & 18 & 3 & 20 \\
\hline LS.3 & 26 & 16 & 17 \\
\hline SSP & 65 & 63 & 59 \\
\hline CS & 172 & 262 & 116 \\
\hline ND.1 & 21 & 12 & 18 \\
\hline ND. 2 & 19 & 16 & 22 \\
\hline ND.3 & 30 & 26 & 31 \\
\hline IBS & 48 & 30 & 45 \\
\hline LCR & 14 & 9 & 18 \\
\hline RM.1 & 2 & 3 & 7 \\
\hline RM. 2 & 163 & 168 & 128 \\
\hline RM.3 & 157 & 156 & 139 \\
\hline AJB.1 & 1 & 3 & 9 \\
\hline AJB. 2 & 1 & 7 & 17 \\
\hline AJB. 3 & 4 & 0 & 12 \\
\hline Média & 29,5 & 27,6 & 26,4 \\
\hline
\end{tabular}


Tabela 3. Concentração absoluta de células CD34+ em amostras de produto de aférese pelos três métodos de análise

\begin{tabular}{|c|c|c|c|}
\hline $\begin{array}{l}\text { Identificação } \\
\text { Pac. / no Aférese }\end{array}$ & $\begin{array}{l}\text { P. Molhouse mod. } \\
\text { células } / \mathrm{mm}^{3}\end{array}$ & $\begin{array}{l}\text { Prot. ISHAGE } \\
\text { células } / \mathrm{mm}^{3}\end{array}$ & $\begin{array}{l}\text { Imagn } 2000 \\
\text { células } / \mathrm{mm}^{3}\end{array}$ \\
\hline $\mathrm{AK}$ & 13276 & 10962 & 14360 \\
\hline MSGC.1 & 980 & 588 & 1344 \\
\hline AGF.1 & 612 & 119 & 485 \\
\hline MSGC. 2 & 169 & 176 & 120 \\
\hline AGF.2 & 70 & 70 & 97 \\
\hline AGF.3 & 13 & 11 & 28 \\
\hline EB.1 & 26 & 1 & 30 \\
\hline EB. 2 & 59 & 81 & 33 \\
\hline LBS.1 & 903 & 780 & 876 \\
\hline LBS. 2 & 655 & 917 & 792 \\
\hline LBS.3 & 1658 & 608 & 1356 \\
\hline SSP.1 & 2566 & 2068 & 1792 \\
\hline SSP. 2 & 2700 & 5556 & 5256 \\
\hline LBS. 4 & 584 & 716 & 584 \\
\hline LBS. 5 & 875 & 554 & 722 \\
\hline SSP. 3 & 4503 & 3591 & 5170 \\
\hline JTJ & 1862 & 1280 & 1862 \\
\hline IW.1 & 1827 & 1512 & 1796 \\
\hline IW.2 & 1942 & 1145 & 1539 \\
\hline JRPJ & 1006 & 1243 & 1398 \\
\hline MNGA.1 & 771 & 638 & 830 \\
\hline MNGA. 2 & 878 & 745 & 1000 \\
\hline MNGA.3 & 1040 & 885 & 1010 \\
\hline MLCSC & 66 & 52 & 62 \\
\hline AVP.1 & 72 & 72 & 64 \\
\hline AVP. 2 & 148 & 36 & 74 \\
\hline IBS.1 & 4640 & 4355 & 4760 \\
\hline IBS.2 & 1940 & 1777 & 1558 \\
\hline GG.1 & 4019 & 4111 & 4104 \\
\hline GG.2 & 4973 & 5023 & 5268 \\
\hline $\mathrm{AK}$ & 13276 & 10962 & 14360 \\
\hline Média & 2197,1 & 1955,9 & 2346,1 \\
\hline
\end{tabular}


Tabela 4. Concentração absoluta de células CD34+em amostras de sangue de cordão umbilical pelos três métodos de análise

\begin{tabular}{|l|c|c|c}
\hline $\begin{array}{l}\text { Identificação } \\
\text { Pac. / no Aférese }\end{array}$ & $\begin{array}{c}\text { P. Mulhouse mod. } \\
\text { células/mm }\end{array}$ & $\begin{array}{c}\text { Prot. ISHAGE } \\
\text { células/mm }\end{array}$ & $\begin{array}{c}\text { Imagn 2000 } \\
\text { células/mm }\end{array}$ \\
\hline SCU 11 & 45 & 81 & 72 \\
\hline PC 3 & 16 & 24 & 25 \\
\hline R.T. & 93 & 92 & 84 \\
\hline PC 5 & 27 & 31 & 60 \\
\hline PC 6 & 14 & 17 & 21 \\
\hline PC 7 & 43 & 49 & 53 \\
PC 8 & 19 & 18 & 27 \\
PC 9 & 48 & 53 & 50 \\
\hline PC 10 & 40 & 45 & 47 \\
\hline PC 11 & 62 & 66 & 57 \\
\hline PC 12 & 50 & 43 & 45 \\
PC 13 & 16 & 20 & 27 \\
PC 14 & 58 & 54 & 52 \\
\hline PC 15 & 16 & 25 & 23 \\
\hline PC 16 & 44 & 26 & 40 \\
\hline PC 17 & 44 & 40 & 45 \\
\hline PC 18 & 28 & 31 & 57 \\
GUCR & 28 & 23 & 26 \\
\hline PC 24 & 35 & 24 & 49 \\
\hline PC 25 & 26 & 14 & 26 \\
\hline Média & 37,6 & 38,8 & 44,3 \\
\hline
\end{tabular}

\section{Análise dos Resultados}

A aplicação do teste $\mathrm{T}$ de Student e de Mann Withney para analisar as diferenças entre os três métodos nos três tipos de materiais não evidenciaram diferenças significativas $(\mathrm{p}<0,05)$. A correlação entre os métodos está representada na Tabela 5.

Tabela 5. Diferenças entre os métodos realizados

\begin{tabular}{l|l|l|l}
\hline Coeficiente de Correlação & $\begin{array}{l}\text { Sangue } \\
\text { Periférico } \\
\mathrm{n}=32\end{array}$ & $\begin{array}{l}\text { Produto } \\
\text { de Aférese } \\
\mathrm{n}=31\end{array}$ & $\begin{array}{l}\text { Cordão } \\
\text { Umbilical } \\
\mathrm{n}=20\end{array}$ \\
\hline P. Mulhouse mod. x Prot. ISHAGE & $\mathrm{R}^{2}: 0,93$ & 0,95 & 0,86 \\
Prot. ISHAGE x Imagn 2000 & $\mathrm{R}^{2}: 0,96$ & 0,98 & 0,80 \\
\hline P. Mulhouse mod. x Imagn 2000 & $\mathrm{R}^{2}: 0,91$ & 0,98 & 0,86 \\
\hline
\end{tabular}




\section{Discussão}

\section{Precisão das Metodologias}

A precisão da metodologia em uso no Laboratório do HIAE tem oscilado entre 10 a 15\%, de maneira similar à literatura quando analisada a variabilidade intralaboratorial $(6,16)$. Essa precisão foi similar na avaliação realizada para este trabalho nas três metodologias adotadas. A grande variabilidade interlaboratorial decorrente da ausência de padronização metodológica para a determinação de CD34; da variabilidade de classes de anticorpos monoclonais disponíveis $(25,26)$ e por inúmeros outros fatores interferentes tem sido muito ampla. Nos primeiros levantamentos do UK NEQAS - United Kingdom, a variação ultrapassa 100\% e com a evolução na padronização técnica, hoje já é inferior a $25 \%$ para os participantes deste sistema (21). Certamente a adoção de maiores padronizações metodológicas como as propostas do ISHAGE e outras $(17,27)$, a disponibilidade de programas de proficiência interlaboratorial (UK NEQAS e outros) possibilitam uma redução ainda maior dessa variabilidade, mas que dificilmente será inferior aos 10\% obteníveis atualmente em laboratórios únicos. Essa limitação é inerente à metodologia de quantificar eventos raros, como discutido na bibliografia $(19,28)$ e dificilmente será superada, como ainda não o foi para a própria quantificação de leucócitos totais, mesmo nos contadores automatizados mais modernos.

\section{Comparação das Metodologias}

Nossos dados demonstram não existir diferenças estatisticamente significantes entre os três métodos analíticos estudados para a quantificação de células CD34+ em amostras de sangue periférico; concentrados de leucoaféreses e sangue de cordão umbilical. A correlação entre os três métodos está dentro dos limites aceitáveis, validando as três metodologias para essa quantificação nesses diferentes tipos de amostra.

No entanto, a observação dos resultados, amostra à amostra, revela diferenças maiores de 100\% entre as metodologias de forma aleatória entre elas a cada amostra divergente, afastando um erro sistemático (de método, equipamento ou calibração). Essas diferenças isoladas podem ser explicadas pela heterogeneidade do material, interferências próprias de uma amostra em um método e limitações da própria metodologia representada pelo seu coeficiente de variação.

As diversas recomendações têm sido investigadas e publicadas como: isolamento de leucócitos pela lise de eritrócitos (22), discriminação de células viáveis (23); o uso de anticorpo monoclonal CD34 classe III com afinidade à todas as variantes glicosiladas da molécula, assim como o uso do fluorocromo ficoeritrina (PE) com possibilidade de discriminação maior da autofluorescência na amostra, seu maior tamanho de molécula com menor afinidade inespecífica aos receptores $\mathrm{Fc}$ e as células mortas (15, 25, 26, 29, 30); a utilização de CD45 como forma de excluir resíduos eritrocitários, plaquetas e seus agregados (17), mas com limitações ao excluir algumas células CD34+, mas CD45-, possíveis após processo de quimioterapia anterior, dependendo de algumas variantes glicosiladas do CD45. Tem também sido citado na literatura que nos casos de quantificação de CD34+ em sangue de cordão umbilical e outros materiais ricos em células $\mathrm{CD} 34+$ é conveniente à captação (gate) sequencial (15) e o uso de controle isotipo $(11,18)$ do mesmo fabricante para manter a relação fluorocromo/proteína (16).

Os novos métodos que têm sido desenvolvidos procuram numa mesma análise obter a quantificação absoluta de células CD34, evitando o uso de duas determinações (relativa e absoluta) envolvendo dois equipamentos (plataforma dupla) que aumentam teoricamente a variabilidade final do método. Entre esses métodos destacam-se o Imagn 2000 e os desenvolvidos para a citometria de fluxo, Procount (Becton Dickinson) e Stem Kit (Coulter-Immunotech) que pela adição de beads fluorescentes permitem a quantificação em concentração absoluta das células CD34 positivas. Pelo menos um deles, que testamos nesse trabalho, apesar da simplicidade operacional, não demonstrou vantagens significantes na precisão metodológica. 
Efeito da Precisão Metodológica na aplicação clínica da quantificação de células CD34+

O coeficiente de variação mínimo de 10 a 15\% das metodologias de quantificação de células CD34+ e as variações aleatórias intra e interlaboratoriais de até 100\% refletem-se na utilização dessa metodologia para a avaliação de viabilidade de um material coletado por aférese ou de sangue de cordão para a realização do procedimento de recuperação da hematopoese após ablação terapêutica da medula óssea (34). Também influencia significativamente as análises de tempo de recuperação e incidência de GVHD selecionados ao número e tipos de células CD34+ infundidas.

Tomando-se como exemplos as diferenças obtidas nos casos SSP.2 e LBS.3 para as três metodologias, estabeleceu-se como volume final padrão do produto da aférese $60 \mathrm{ml}$ e o peso do paciente receptor de $70 \mathrm{~kg}$, teremos os seguintes resultados expressos em células por quilo de peso do receptor (tabela 6).

Tabela 6. Diferenças entre dois casos segundo as metodologias

\begin{tabular}{l|c|c|c|}
\hline CASO & $\begin{array}{l}\text { ISHAGE } \\
\text { células } \\
\text { CD34x10 }\end{array}$ & $\begin{array}{l}\text { Mulhouse mod. } \\
\text { células } \\
\text { CD34x10 }\end{array}$ & $\begin{array}{l}\text { Imagn 2000 } \\
\text { células } \\
\text { CD34x10 }\end{array}$ \\
\hline SSP.2 & 4,7 & 2,3 & 4,5 \\
LBS.2 & 0,5 & 1,4 & 1,1 \\
\hline
\end{tabular}

Essa variabilidade está representada nas indicações dos diversos serviços e na literatura $(10,31,32,33)$, que colocam os valores mínimos desde 0,2 até 2,0 células CD $34+\times 10^{6}$ por quilo e admitem como valor ideal 1,0 ate 2,0. Esses fatos dificultam a decisão caso a caso, quando se deve ou não realizar o procedimento diante do número de células disponíveis, quantas plasmaféreses realizar a mais para obter um número "ideal" com significante aumento de custo e todas as avaliações de relacionamento matemático entre número de células com o tempo para recuperação de indicadores hematológicos, que tem reflexo direto na incidência de complicações, tempo e consequentemente com o custo da hospitalização desse tipo de paciente.

\section{Conclusão}

Foram quantificadas células CD34+ em sangue periférico após estímulo com G-CSF, concentrados de aféreses e sangue de cordão umbilical pelos métodos de citometria de fluxo, usando o protocolo ISHAGE, protocolo Mulhouse modificado e pelo citômetro de volume fixo e plataforma única Imagn 2000.

Os resultados não apresentaram diferenças analíticas estatisticamente significantes e os coeficientes de correlação foram para sangue periférico, produto de aférese e cordão umbilical respectivamente nos protocolos ISHAGE $x$ Mulhouse modificado de 0,93, 0,95 e 0.86; no protocolo ISHAGE x Imagn, respectivamente de 0,96, 0,98 e 0,86 e finalmente no protocolo Mulhouse modificado x Imagn respectivamente de 0,91, 0,98 e 0,86. A precisão encontrada, dos métodos utilizados variou de $11 \%$ a $15 \%$ e os programas de proficiência têm demonstrado a redução progressiva da variação interlaboratorial de mais de $100 \%$ para $25 \%$. As variações do método, as interlaboratoriais e as aleatórias, trazem reflexo na utilização clínica da quantificação de células CD34 seja na adoção de um valor mínimo para a realização dos procedimentos de recuperação da hematopoese após terapêutica ablativa da medula óssea, como também na monitorização dos resultados desse procedimento.

As metodologias analisadas são similares entre si para a quantificação de células tronco em sangue periférico após estimulação com G-CSF; concentrados obtidos por aféreses e sangue de cordão umbilical.

A variação analítica tem coeficiente de variação superior à $10 \%$ e variações aleatórias intra e interlaboratoriais de até 100\% que se refletem no uso clínico dessa metodologia. 


\section{Quantification of $\mathrm{CD} 34+$ cells in peripheral blood, leukapheresis products and cord blood: a comparative study of three different cytometric methodologies}

Nydia S. Bacal, João C. C. Guerra, Eurípedes Ferreira, Sonia T. Nozawa, Ruth H. Kanayama, Daniel Di Pietro, Jacyr Pasternack, Nelson Hamersclack, Luiz G. M. Rosenfeld

\section{Abstract}

CD34+ cell quantification in peripheral blood is useful to determinate the best moment to begin apheresis while, when applied in leukapheresis products and umbilical cord blood it is important to establish the total number of CD34+ in stem cell transplantation. We aimed at comparing three different methodologies of CD34+ cell quantification. Three different blood products were analyzed: a) peripheral blood samples from 32 patients collected using EDTA, mobilized without previous chemotherapy and with G-CSF 50 $\mathrm{mcg} / \mathrm{Kg} /$ total. b) 31 leukapheresis samples obtained using a CS 3000 (Baxter) cell separator. c) 20 cord blood samples collected using umbilical venipuncture. All samples were maintained at room temperature and analyzed until $24 \mathrm{~h}$ after collection by three different methods: double platform protocols ISHAGE, a Mulbouse modified (for analysis of a higher number of events) applied in a Epics $X L$ flow cytometer (Coulter) and a single platform for a fixed volume cytometer IMAGN 2000 (Biometric Imaging) according to manufacturer instructions. Monoclonal antibodies used were: CD 45-FITC, CD 34-PE and IgG1-PE isotype control (Immunotech, Marseille, FR). Statistical analysis was made using ANOVA and T Student test. Results showed no statistical differences $(p<0.05)$ among the three methods used to quantify CD34+ cells from any sample source.

Rev.bras.hematol.hemoter., 2001, 23(2): 69-78

Key words: Flow cytometry, volume fixed cytometry, CD34+ quantification, double platform, single platform

\section{Referências Bibliográficas}

1. Bender J., To LB., Williams S., Schwartzberg L. Defining a therapeutic dose of peripheral blood stem cells. J. Hematotherapy. 1992; 1: 329-342.

2. Davis BH., Foucar K., Szczarkowski W., et al. U.S. Canadian Consensus Recommendations on the Immunophenotypic Analysis of Hematologic Neoplasia by Flow Cytometry: Medical Indications. Cytometry. 1997; 30: 249-263.

3. Scott M.A. In search of the haematopoietic stem cell. British Journal of Haematology. 1995; 90: 738-743.

4. Sutherland D.R., Keating A., Nayar R., et al. Sensitive detection and enumeration of CD34 cells in peripheral and cord blood by flow cytometry. Experimental Hematology. 1994; 22: 1003-1010.

5. Gluckman E., Rocha V., Boyer-Chammard A. et al. For the Eurocord Transplant Group and the European Blood and Marrow Transplantation Group. Outcome of cordblood trasplantation from related and unrelated donors. N.Eng1.J.Med. 1997; 337: 373-381.

6. Hollingsworth K.L, Zimmerman T.M., Karrison T. et al. The CD $34+$ cell concentration in peripheral blood predicts CD34+ cell yield in the leukapheresis product. Cytotherapy. 1999; 1 (2): 141-146.

7. Weeks F.M., Yee G.C., Bartfield A.A. et al. The true cost of bone marrow transplantation. The American Journal of the Medical Sciences. 1997; 314 : 101-112.

8. Duncan N., Hewetson M., Powles R. et al. An economic evaluation of peripheral blood stem cell transplantation as an alternative to autologous bone marrow transplantation in Multiple Myeloma. Bone Marrow Transplantation. 1996; 18(6): 1175-8.

9. Wendel RF, Lazar A., Melges S. et al. The absolute number of circulating CD $34+$ cells as the best predictor of peripheral hematopoietic stem cell yield. Journal of Hematotherapy. 1999; 8: 255-62.

10. Schots R., Van Riet I., Damiaens S et al. The absolute number of circulating CD $34+$ cells 
predicts the number of hematopoietic stem cells that can be collected by apheresis. Bone Marrow Transplantation. 1996; 17: 509515.

11. Siena S., Bregni M., Belli N. Flow Cytometry for clinical estimation of circulating bematopoietic progenitors for autologous transplantation in cancer patients. Blood. 1991; 77: 400.

12. Schuvella N., Siegert W., Beyer J. et al. Autografting with blood progenitor cells: predictive value of preapheresis blood cell counts on progenitor cell harvest and correlation of reinfused cell dose with bematopoietic reconstituion. Ann Hematology. 1995; 71: 227-234.

13. Mavroudis D., Read E., Cottler-Fox M. et al. CD34+ cell dose predicts survival, postransplant morbidity and rate of hematologic recovery after allogeneic marrow transplants for hematologic malignancies. Blood. 1996; 88(8): 32233229 .

14. Woronoff-Lemsi M.C., Arveux P., Limat S. et al. Cost comparative study os autologous peripheral blood progenitor cells (PBPC) and bone marrow (ABM) transplantations for nonHodgkin's lymphoma patients. Bone Marrow Transplantation. 1997; 20: 975-82.

15. Gratama J.W., Orfao A., Barnett D. et al. Flow Cytometric enumeration of CD34+hematopoietic Stem and Progenitors cells. Cytometry 1998; 34: 128-142.

16. Marti G., Johnsen H., Sutherland D.R. et al. $A$ convergence of method for a worldwide standard for CD34+ cell enumeration.

Journal of Hematotherapy. 1998; 7: 105109.

17. Sutherland D.R., Anderson L., Nayar M.K.R. et al. The ISHAGE guidelines for CD34+ cell determination by flow cytometry. $\mathbf{J}$. Hemothr. 1996; 5: 213-26.

18. Bender J.G., Unverzagt K., Walker D. Guidelines for determination of CD34+ cells by flow cytometry application to the harvesting and transplant ation of peripheral blood stem cell. In : Hematopoietic Stem Cells The Mulhouse Manual, Wunder E., Sovalat H, Henon PR, Serke S (eds) Alpha Med
Press, Dayton OH 1994; pp 31-43.

19. Wunder E., Sovalat H., Fritsch G. et al. Report on the European Workshop on peripheral blood stem cell determination and standardization-Mulbouse. Journal of Hematotherapy. 1992; 1: 131-142.

20. Dietz L.J., Dubrow R.S., Manian B.S. et al. Volumetric capillary cytometry. A new method for absolut cell enumeration. Cytometry. 1996; 23: 177-186.

21. Barnett D., Granger V., Storie I. et al. Quality assessment of $\mathrm{CD} 34+$ stem cell enumeration : experience of the United Kingdom National External Quality Assessment Scheme (UK NEQAS) using a unique stable whole blood preparation. British Journal of Hematology. 1998; 102: 553-565.

22. Bossuyt X., Marti G.E., Fleisher T.A. Comparative Analysis of Whole Blood Lysis Methods for Flow Cytometry. Cytometry. 1997; 30: 124-133.

23. Gross H.J., Verwer B., Houck D. et al. Detection of rare cells at a frequency of one per million by Flow Cytometry. Cytometry. 1993; 14: 519-526.

24. Schmid I., Krall W.J., Uittenbogaart C.H. et al. Dead cell discrimination with 7-amino actinimycin D in combination with dual color immunofluorescence in single laser flow cytometry. Cytometry. 1992; 13: 204-8.

25. Serke S., Huhn D. Expression of Class I, II and III Epitopes of the CD34 Antigen by normal and leukemic hemopoietic cells. Cytometry. 1996; 26: 154-160.

26. Steen R., Tjonnfjord G.E., Gaudernack G. et al. Differences in the distribution of CD34 epitopes on normal haemopoietic progenitor cells and leukaemic blast cells. British Journal of Haematology 1996; 94: 597-605.

27. Keeney M., Chin-Yee I., Weir K. et al. Single platform Flow Cytometric absolute CD34+ cell counts based on the ISHAGE guidelines. Cytometry 1998; 34: 61-70.

28. Johnsen H.E. Report from a Nordic Workshop on CD34+ cell analysis: technical recommendations for Progenitor Cell Enumeration in leukapheresis from Multiple Myeloma patients. Journal of Hematotherapy. 1995; 4: 21-28. 
29. Krause S.D., Fackler M.J., Civin C.I., May W.S. CD34: Structure, Biology and Clinical Utility. Blood. 1996; 87: 1-13.

30. To L.B., Haylock D.N., Simmons P.J. et al. The biology and clinical uses of blood stem cells. Blood. 1997; 89: 2233-2258.

31. Tricot G., Jagannath S., Vesole D. Peripheral blood stem cell transplants for multiple myeloma: identification of favorable variables for rapid engraftment in 225 patients. Blood. 1995; 85: 588-596.

32. Weaver C.H., Potz J., Redmond J. et al. Engraftment and outcomes of patients receiving myeloablative therapy followed by autologous peripheral blood stem cells with a low CD34+ cell content. Bone Marrow Transplantation. 1997; 19: 1103-1110.

33. Zimmerman T.M., Lee W.J., Bender J.G., Mick R. et al. Quantitative CD34 analysis may be used to guide peripheral blood stem cell harvests. Bone Marrow Transplantation. 1995; 15: 439-44.

34. Cairo M.S.C., Wagner J.E. Placental and/ or Umbilical Cord Blood: an Alternative ource of Hematopoietic Stem Cells for Transplantation. Blood. 1997; 90 (12): 4665-78.

Recebido: 12/06/01

Aceito: 20/07/01 